\title{
Taking the lead on climate change: modelling and monitoring the fate of an Amazonian frog
}

\author{
Elodie A. Courtois, Elodie Michel, Quentin Martinez, Kevin Pineau \\ Maël Dewynter, Gentile F. Ficetola and Antoine Fouquet
}

\begin{abstract}
Climate change is expected to have important impacts on biodiversity. However, cases showing explicit links between species decline and climate are scarce, mostly because of a lack of baseline data. Tropical ectotherms with narrow altitudinal ranges are particularly sensitive to climate change; for example the frog Pristimantis espedeus may be at risk, with only nine populations known to date in French Guiana, all on isolated massifs. Ecological niche modelling indicated that these populations could disappear by 2070. To facilitate testing of this prediction we conducted a study to design an efficient, cost-effective monitoring protocol, combining occupancy rate estimations using passive acoustic recorders, and abundance estimations using acoustic repeated counts and capture-mark-recapture. We found the passive recorders to be effective, with a detection probability of 0.8 . Two recording sessions were sufficient to estimate occupancy rates reliably. A minimum of 57 surveyed sites were required to detect a decline of $15 \%$ in occupancy between two consecutive monitoring events. Acoustic repeated counts and capture-mark-recapture yielded similar density estimates ( 1.6 and 1.8 calling males per $100 \mathrm{~m}^{2}$, respectively). Based on these results we present a protocol based on passive acoustic recording and abundance monitoring to monitor $P$. espedeus populations.
\end{abstract}

Keywords Altitude, Amazonia, amphibian decline, climate change, conservation, French Guiana, Neotropics, Pristimantis espedeus

Elodie A. Courtois (Corresponding author), Elodie Michel, Quentin Martinez and ANTOINe Fouquet CNRS Guyane USR 3456, Immeuble Le Relais, 2 Avenue Gustave Charlery, 97300 Cayenne, French Guiana

E-mail courtoiselodie@gmail.com

Kevin Pineau Groupe d'Etude et de Protection des Oiseaux de Guyane, Cayenne, French Guiana

MaËL Dewynter Biotope Guyane, Cayenne, French Guiana

Gentile F. Ficetola Laboratoire d'Ecologie Alpine, Université Joseph Fourier, Grenoble, France

Received 13 August 2014. Revision requested 27 October 2014.

Accepted 20 January 2015. First published online 1 June 2015.

\section{Introduction}

Climate change will be one of the most significant $\checkmark$ threats to biodiversity in the future (Travis, 2003), and some studies suggest that climate-driven extinctions and range reductions are already widespread (Walther et al., 2002; Parmesan \& Yohe, 2003; Thomas et al., 2006). Species vary in their resilience to climatic change. Climate change is expected to have greater impact in the tropics (Foden et al., 2013) given that tropical species often have narrower thermal tolerances than temperate species (Sheldon et al., 2011; Sunday et al., 2011). This is particularly true for ectotherms such as amphibians (Snyder \& Weathers, 1975; Buckley et al., 2012; Duarte et al., 2012) because their basic physiology is dependent on environmental temperature and humidity (Blaustein \& Wake, 1990; Sheldon et al., 2011).

Latitudinal and altitudinal range shifts that are probably attributable to climate change have already been reported for several amphibians (Houlahan et al., 2000; Stuart et al., 2004), with other contributing factors including pathogenic diseases and habitat loss (Pounds et al., 2006). Among these species, those associated with tropical mountainous habitats seem to be particularly vulnerable. These species often have a restricted distribution (Stuart et al., 2004; Pounds et al., 2006), which is a further cause for concern. Nonetheless, unambiguous evidence of climate change-driven decline is scarce (Cahill et al., 2013) for a number of reasons: the absence of baseline data, the difficulty in detecting declines in abundance of rare or elusive species, and the difficulty in linking these declines unambiguously to changes in climate (Thomas et al., 2006). A pertinent choice of study species, a combination of various methods, and long-term monitoring of species and environmental parameters are necessary to overcome these limitations.

Pristimantis espedeus (Amphibia, Craugastoridae) is a recently described arboreal frog species (mean snout-vent length $=23 \pm S D 1.1 \mathrm{~mm}$ for adult males) belonging to the Terrarana, a species-rich neotropical clade (Hedges et al., 2008) characterized by direct development, with no free aquatic larval stage. Females are elusive (only one female has been collected so far) but males are easily detectable by their conspicuous calls at dusk and dawn (Fouquet et al., 2013). A large proportion of species in this clade occur within narrow altitudinal ranges, rendering them particularly 

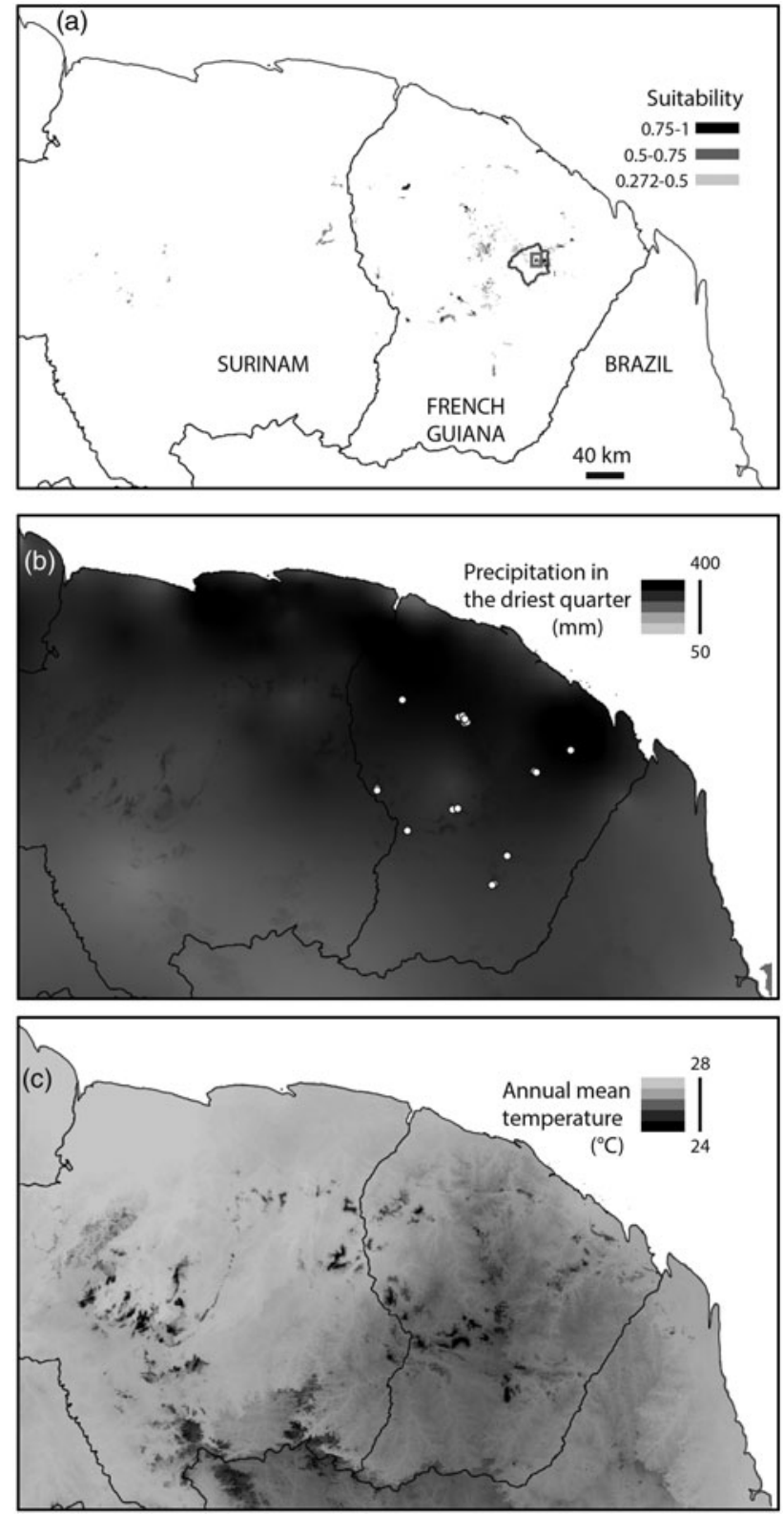

FIG. 1 (a) Habitat suitability for Pristimantis espedeus in the study area under current climatic conditions, based on Maxent analysis. The rectangle indicates the location of the Balenfois Massif within the Nouragues Reserve. (b) Distribution of precipitation in the driest quarter. The white dots indicate locations of $P$. espedeus used in ecological niche modelling.

(c) Distribution of annual mean temperature.

sensitive to climate change. Shifts in altitudinal distribution have been observed in the Terrarana, potentially related to changes in precipitation or increasing temperature (Pounds et al., 1999; Burrowes et al., 2004). Pristimantis espedeus is known to occur only in nine locations, all in French Guiana on isolated massifs of $>400 \mathrm{~m}$ altitude, and is not known to occur below $200 \mathrm{~m}$ (Fouquet et al., 2013). This species therefore represents a suitable model for investigation of the impact of climate change on tropical amphibians.
Ecological niche modelling facilitates identification of relationships between occurrences of a species and environmental or physical variables (Franklin, 2009; Warren, 2012). Combining this with future climate projections could therefore facilitate the assessment of the vulnerability of a population to climate change (Lemes \& Loyola, 2013; Nori et al., 2013). Monitoring could then be used to validate the model predictions. Such empirical testing of the predictive power of ecological niche modelling is rare (Dawson et al., 2011). It requires long-term monitoring and thus efficient and cost-effective methods. The choice of monitoring protocol (capture-mark-recapture, Stanley \& Burnham, 1999; repeated counts, Royle, 2004; or site occupancy, MacKenzie et al., 2002) depends on the availability of time and funding, and on the species' biology (Joseph et al., 2006).

We used ecological niche modelling to evaluate how the populations of $P$. espedeus could potentially respond to climate change by 2070 , and conducted a pilot study on a population of $P$. espedeus to design an efficient and costeffective monitoring protocol. We combined estimation of occupancy rates using passive acoustic recorders and abundance estimation using acoustic repeated counts and capture-mark-recapture. These estimates were used to (1) identify the parameters (e.g. month, time of day) influencing the detection probability of the species, and determine the numbers of recording sessions and sites that would be necessary for long-term monitoring, and (2) test whether the use of acoustic repeated counts provides a reliable estimation of the abundance of calling males. Based on this pilot study we provide recommendations for the implementation of a long-term monitoring protocol for P. espedeus.

\section{Study area}

The pilot monitoring study was conducted at the Nouragues Biological Research Station on the Balenfois Massif, in the Nouragues Natural Reserve in French Guiana (Fig. 1). Mean annual temperature is $26^{\circ} \mathrm{C}$ and annual rainfall is c. $3,000 \mathrm{~mm}$, with a rainy season during January-June (Bongers et al., 2001).

\section{Methods}

\section{Ecological niche modelling}

We used the Maxent (maximum entropy) algorithm to model the current and projected environmental suitability for P. espedeus. This approach is based on presence data (records of the species) and it estimates environmental suitability on a pixel-by-pixel basis (Phillips et al., 2006). Suitability is calculated by minimizing the relative entropy between the probability densities of the landscape covariates with and without species presence (Elith et al., 2011). 

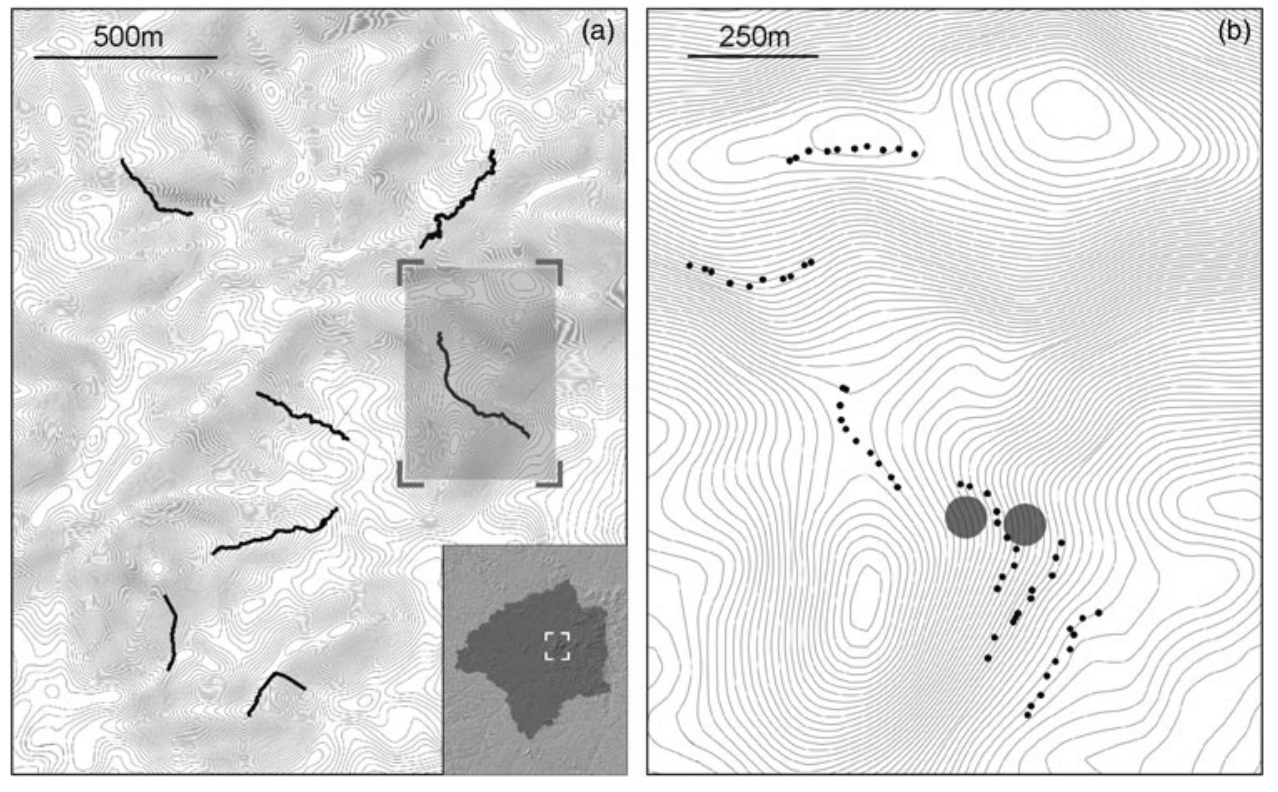

FIG. 2 (a) Location of the seven transects used for the presence/absence protocol. The rectangle on the inset indicates the location of the main map in the Nouragues Natural Reserve, and the shaded rectangle indicates the location of (b). (b) The six transects, with 10 calling survey sites on each transect, used for the abundance protocol; the grey circles indicate the patches used for the capture-mark-recapture protocol.
We identified 21 occurrence sites for the species on nine massifs in French Guiana. We obtained current climate data and a projected climate scenario for 2070 (HadGEM2ES; Moss et al., 2010) from the WorldClim database. These data were used at a 30-arc-second spatial resolution and cropped to the coverage of the eastern Guiana Shield (Fig. 1). We used four variables relevant to the species' association with higher elevation, and its direct development and reproduction during the rainy season $(\mathrm{ALT}=$ elevation, $\mathrm{BIO} 1=$ annual mean temperature, $\mathrm{BIO} 12=$ annual precipita tion and $\mathrm{BIO}_{17}=$ precipitation in the driest quarter). All correlations between these variables are $<0.4$. We selected the logistic output format because it is robust even when species prevalence is unknown, and provides an index of suitability given the constraints imposed by environmental variables (Phillips et al., 2006). In this case, grid cells with small logistic values were predicted to be unsuitable for the studied species given the ecological niche determined from maximum entropy analysis with actual climatic variables. We used a random sample of 10,000 sites from the study area as background data to represent the distribution of environmental conditions in the area. We used the 1oth percentile training presence as a suitability threshold (i.e. we assumed that a cell was suitable if its suitability score computed by Maxent was in the 1oth percentile of training presence points). The 1oth percentile threshold is considered to have good predictive capacity (Pearson et al., 2007).

We used cross-validation to evaluate the robustness of Maxent models. For this, we ran the model five times, each time using a different group of cases, using $80 \%$ of presences as training points and the remaining $20 \%$ as test points. We then evaluated the performance of models by comparing areas under the receiver operating characteristic curve, which measures the quality of a ranking of sites, and calculating the correlation among suitability maps obtained using the five training models and using the complete dataset. We then used the Maxent model to estimate suitable area for the species under projected future conditions.

\section{Presence/absence protocol}

Site occupancy models are particularly well adapted for tracking changes in a species' distribution pattern or altitudinal range (MacKenzie et al., 2002). They can be used to estimate the detection probability for a given species (i.e. the probability of detecting a species at a site, knowing that it is present) and determine its occupancy rate (i.e. the proportion of the site occupied by the species).

We defined seven transects along altitudinal gradients (i.e. along the slope of the massif; Fig. 2c), with five recording sites at fixed elevations (130, 180, 230, 280 and $330 \mathrm{~m}$ ) on each. There were four recording sessions, in 2013 (23 January-14 February, 14 February-18 March, 18 March-8 April, 13-30 June). During each recording session we placed a Song Meter SM2 digital audio field recorder, equipped with a horizontally oriented omnidirectional microphone, at a height of c. $1.5 \mathrm{~m}$ for 3 consecutive days at each site on a transect. Recorders were programmed to record during 05.00-07.00 and 17.30-19.30 each day, to coincide with the calling activity of $P$. espedeus (Fouquet et al., 2013). Thus six recordings were made at each recording site in each session (three at dawn and three at dusk). Recordings were split into 40 3-minute files, using Slice Audio File Splitter v. 2.o (NCH Software, Greenwood Village, USA). The first $20 \mathrm{~s}$ of each recording were visualized as sonograms using Raven Lite 1.o (Charif et al., 2006), and these were used to detect the 
characteristic call of male $P$. espedeus (2-5 notes of dominant frequency 2.5-3.5 KHz; Fouquet et al., 2013).

During each recording session two HOBO data loggers (Onset Computer Corporation, Bourne, USA) recorded ambient temperature and light intensity every 4 minutes. We calculated the total light intensity and the minimum temperature during the day for 8 days on each transect. We tested whether minimum daily temperature differed among recording sites (i.e. at each elevation) using ANOVA. Other confounding factors (e.g. sun exposure, slope) may also affect minimum temperature but we did not have sufficient data to include them in the analysis.

Detection/non detection histories (for 35 recording sites, sampled 24 times each) were used to determine detection probability and occupancy rates (MacKenzie et al., 2002). First, we tested whether the session (factor with four levels, $\mathrm{S}_{1}-\mathrm{S}_{4}$ ) or the recording period (factor with two levels: dusk or dawn) had an impact on the detection probability of the species. For this, we compared the Akaike information criterion of the null model (constant detection probability) with models taking into account session, recording period, and their interaction.

To refine the time interval when detection probability is highest, we split each 2-hour recording session into eight 15minute segments and constructed a second detection/non detection history per segment. We then compared a model taking into account the effect of the time segment with the null model (constant detection probability).

We tested whether the occupancy rate varied with elevation by comparing a model taking into account the effect of elevation on the occupancy rate with the null model (detection probability and occupancy rate constant). For each test we used the Akaike information criterion to select among competing models (Akaike, 1974). All analyses were carried out using the package unmarked (Fiske \& Chandler, 2011) in $R$ v. 2.15.3 (R Development Core Team, 2014).

\section{Protocol for abundance of calling males}

We defined five additional transects, at fixed elevations (130, 180, 230, 280 and 330 m; Fig. 2d). Each comprised 10 calling survey sites at 30-m intervals (Fig. 2d). We estimated the maximum detection distance of calling males was 15 $\mathrm{m}$. We walked each transect six times during 22 January15 April 2013, only under good audio conditions (no rain). If it began to rain during a session, we discarded the data from sites surveyed under heavy rain ( 5 of 36 walks). We undertook each walk during 18.30-19.00, stopping for 2 minutes at each site to estimate the number of calling males. We analysed these repeated count data using Royle's N-mixture models (Royle, 2004) implemented in unmarked in R (Fiske \& Chandler, 2011). These models use spatial replication of repeated count data to estimate the site-specific number of individuals $(\mathrm{N})$, and can be used to obtain a proxy of population abundance (Royle, 2004). Spatial replications at more than one site provide information on the distribution of $\mathrm{N}$, and reasonable estimates of abundance even from scarce data (Royle, 2004). The abundance of each species at each site is assumed to remain constant during the survey, and site-specific abundances are assumed to follow a Poisson or negative binomial distribution. For three of five transects $(180,230$ and $280 \mathrm{~m})$ the Poisson distribution fit the data well (goodness of fit, $\mathrm{P}=0.05, \quad 0.05$ and 0.29 , respectively), and neither a Poisson nor a negative binomial distribution fit the data from the transects at 330 and $380 \mathrm{~m}$ (goodness of fit, $\mathrm{P}<$ 0.01). We therefore used a Poisson distribution for all transects, keeping in mind that the results from transects 330 and $380 \mathrm{~m}$ need to be considered carefully. To test the number of walks necessary for an accurate estimation of the number of calling males at one site, we ran simulations with an increasing number of walks (from 3 to 6). For each simulation a sample of j walks was selected randomly 50 times; we estimated the number of calling males for $\mathrm{j}$ walks and compared it with the estimation from the maximum number of walks (6).

We compared estimates derived from repeated counts with those of the capture-mark-recapture method. Although capture-mark-recapture is the most reliable method for monitoring populations over time (Joseph et al., 2006), it often yields unrealistic results in cases of low species density (Courtois et al., 2013) and it requires a significant investment of time and money (Joseph et al., 2006). Capture-mark-recapture was undertaken within two patches $\left(600 \mathrm{~m}^{2}\right.$ at $220 \mathrm{~m}$ and $1,350 \mathrm{~m}^{2}$ at $240 \mathrm{~m}$; Fig. 2d) located next to the transects used for repeated counts. In each patch we gathered data on four occasions (once per day on 4 consecutive days) in early February 2013. All calling males encountered were captured, and photographed dorsally and laterally for individual identification. We performed closedcapture analysis to estimate abundance and individual detection probability (p-hat), using CAPTURE implemented in MARK (White \& Burnham, 1999). Given the short sampling period, and because the movement capability of $P$. espedeus is likely to be limited, we assumed that the closure assumption was met (Stanley \& Burnham, 1999).

\section{Power analysis}

Using occupancy rates and detection probabilities estimated in this pilot study, we expect to propose adapted recommendations for surveying P. espedeus (Guillera-Arroita et al., 2010). Firstly, we determined the number of survey visits (K) required to estimate species presence at an occupied site with a probability of 0.95 :

$$
K=\frac{\log \left(1-\mathrm{P}^{\prime}\right)}{\log (1-\mathrm{P})}
$$

where $\mathrm{P}$ is the probability of detection and $\mathrm{P}^{\prime}=0.95$ is the probability of detecting the species at least once. 
TABLE 1 Model selection for presence/absence surveys ( $2 \mathrm{~h}$ sessions and 15-minute periods) and for abundance estimation using repeated count data, with number of parameters, Akaike information criterion (AIC), and $\triangle$ AIC.

\begin{tabular}{|c|c|c|c|c|}
\hline Variable & Models* & $\begin{array}{l}\text { No. of } \\
\text { parameters }\end{array}$ & AIC & $\Delta \mathrm{AIC}$ \\
\hline \multicolumn{5}{|l|}{ Presence/absence, 2 h session } \\
\hline \multirow[t]{3}{*}{ Effect on detection probability } & $\mathrm{P}(S+\mathrm{MD}) \psi()$. & 9 & 417.21 & 0 \\
\hline & $\mathrm{P}(S) \psi()$. & 5 & 427.6 & 10.39 \\
\hline & $\mathrm{P}(\mathrm{MD}) \psi()$. & 3 & 621.65 & 204.44 \\
\hline \multirow[t]{2}{*}{ Effect on occupancy rate } & $\mathrm{P}(.) \psi(E)$ & 6 & 604.44 & 0 \\
\hline & $\mathrm{P}(.) \psi()$. & 2 & 629.39 & 212.18 \\
\hline \multicolumn{5}{|c|}{ Presence/absence, 15 -minute periods } \\
\hline \multirow[t]{2}{*}{ Dawn } & $\mathrm{P}(\mathrm{TS}) \psi()$. & 9 & 3117.22 & 0 \\
\hline & $\mathrm{P}(.) \psi()$. & 2 & 3516.93 & 399.7 \\
\hline \multirow[t]{2}{*}{ Dusk } & $\mathrm{P}(\mathrm{TS}) \psi()$. & 9 & 5681.35 & 0 \\
\hline & $\mathrm{P}(.) \psi()$. & 2 & 8340.7 & 2659.35 \\
\hline \multicolumn{5}{|l|}{ Abundance } \\
\hline \multirow[t]{2}{*}{ Effect on occupancy rate } & $\mathrm{P}(.) \psi(E)$ & 8 & 1660.78 & 0 \\
\hline & $\mathrm{P}(.) \psi()$. & 3 & 1739.14 & 78.36 \\
\hline
\end{tabular}

${ }^{\star}$ S, session; MD, moment of the day; E, elevation; TS, time slot

We used occupancy rates and detection probabilities estimated previously at each elevation (Guillera-Arroita et al., 2010) to optimize the study design for detecting a decline in occupancy rate. We used a design with $\mathrm{S}$ sites sampled $\mathrm{K}$ times (we estimated the number of survey visits (K) that are necessary to detect the species at least once with a probability close to one (0.95) given the detection probability of the species estimated in this study). Based on this number of survey visits $(\mathrm{K})$ we determined the number of sampling sites that would be needed to detect a decline in occupancy rate between two surveys, given a proportional decline (effect size) R. We set the power (i.e. the probability of detecting a decline) at 0.8 . The number of sites required was then defined as

$$
S=\left(f_{1}+f_{2}\right) \times\left(\frac{z_{\alpha / 2}+z_{\beta}}{\Psi_{1}-\Psi_{2}}\right)^{2}
$$

where $\Psi_{1}$ is the initial occupancy rate and $\Psi_{2}$ is the occupancy rate after a proportional change $\mathrm{R}$ defined as $\Psi_{2}=\Psi_{1}\left(1^{\prime} R\right)$. Terms $f_{1}$ and $f_{2}$ are defined as $f_{1}=\Psi_{1}\left(1-\Psi_{1}+F\right)$ and $f_{2}=\Psi_{2}$ $\left(1-\Psi_{2}+F\right)$, with $\mathrm{F}$ tending to zero as the probability of missing the species at occupied sites $\left(1-\mathrm{P}^{\prime}\right)$ tends to zero (Guillera-Arroita et al., 2010). The value $z_{\mathrm{i}}$ corresponds to $\alpha$ and $\beta$ errors. We assessed three effect sizes $(R=5,15$ and $30 \%$ ) using a significance level $\alpha=0.05$. We assumed that detection probability and the number of replicate surveys were the same for both sampling events. For these simulations we used the $R$ code implemented in Guillera-Arroita \& Lahoz-Monfort (2012).

\section{Results}

Niche modelling The most important variable explaining the presence of $P$. espedeus was precipitation in the driest quarter (34.8\% contribution to Maxent model), followed by elevation $(24.9 \%)$, annual mean temperature $(21.9 \%)$ and annual mean precipitation (18.3\%). The area under the receiver operating characteristic curve was high (o.998), suggesting the model had a good fit. The 1oth percentile training presence logistic threshold was 0.318 . Considering this value as a suitability threshold, $0.28 \%$ of the area can be considered suitable for the species (Fig. 2c). Cross-validation indicated that the model was robust. In all the cross-validated models, the area under the curve calculated for the test data was high and similar to that for the training data (range 0.984-0.998). Predicted suitability was similar across all the replicated models ( $r>0.95$ in all pairwise correlations) and between the replicated models and the model considering all the presence sites ( $r>0.95$ in all pairwise correlations). For this reason we used the model based on all the presence sites in subsequent analyses. Projections of climate change under the chosen scenario indicate that a suitable range for the species may not exist by 2070; all known presence sites had suitability values below the threshold (maximum suitability in $2070=0.015$ ).

Variation in detection probability Detection probability remained constant during January-April, both at dawn and dusk, but fell to almost zero in June (Table 1, Fig. 3a, b). Taking into account the first three sessions only (January-April), detection probability was higher at dusk (18.3018.45; $0.882 \pm \mathrm{SD} 0.054)$ than at dawn (06.15-06.30; $0.711 \pm$ SD 0.036 ; Table 1, Fig. $3 c, d)$. The probability of detecting a male in $20 \mathrm{~s}$ during $18.30-18.45$ was 0.55 , whereas it was only 0.15 during $06.15-06.30$. The peaks in activity at dawn and dusk coincided with changes in luminosity (Fig. 3c,d). 

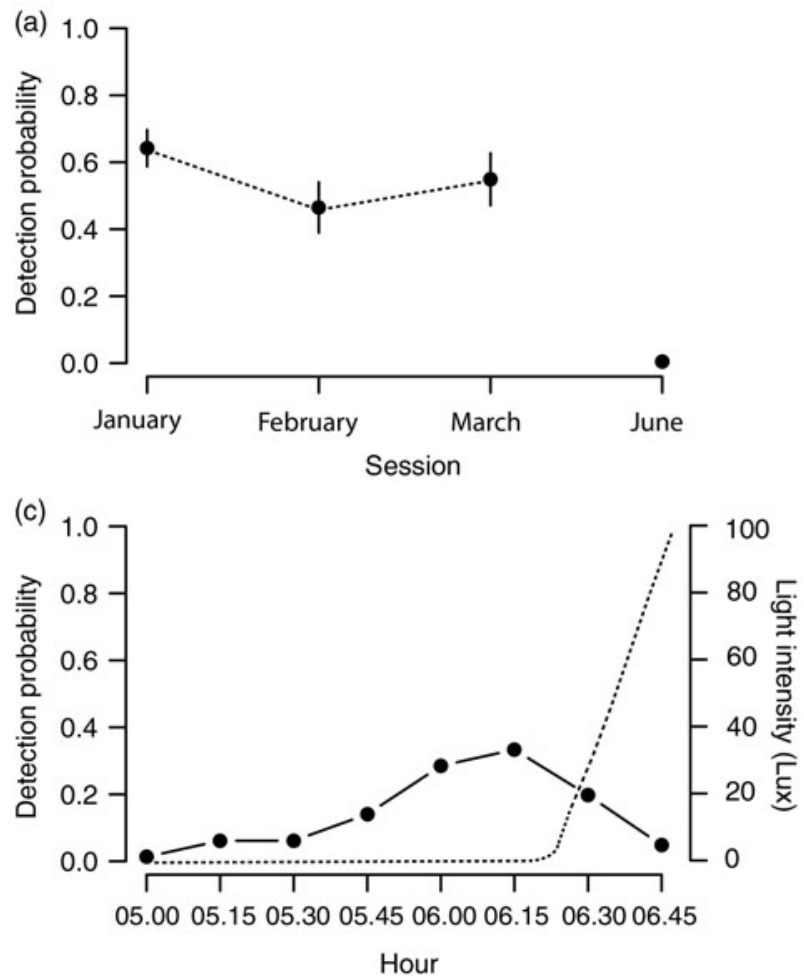
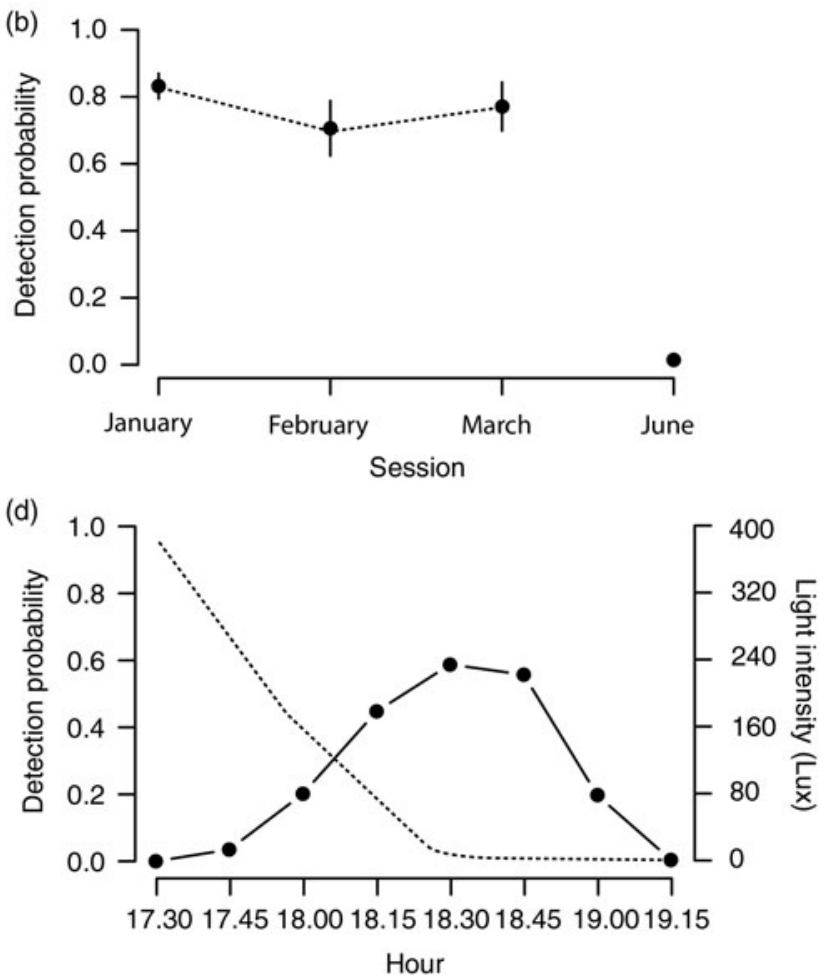

FIG. 3 Variation of detection probability during the four survey sessions at dawn (a) and dusk (b), and variation in detection probability (filled circles) within a 2-hour period at dawn (c) and at dusk (d). In (c) and (d), dashed lines indicate the variation in light intensity averaged across all sites and all transects during the study period.

TABLE 2 Naïve occupancy ( 1 indicates sites where the species as been detected at least once) for each recording site on each transect (T1-T 7 ), with the occupancy rate at each elevation, and the number of sites $(S)$ required to detect a decline, for various changes in occupancy rate $(\mathrm{R})$.

\begin{tabular}{lllllllllrrr}
\hline Altitude $(\mathrm{m})$ & $\mathrm{T} 1$ & $\mathrm{~T} 2$ & $\mathrm{~T} 3$ & $\mathrm{~T} 4$ & $\mathrm{~T} 5$ & $\mathrm{~T} 6$ & $\mathrm{~T} 7$ & Occupancy rate & $S(R=0.05)$ & $S(R=0.15)$ & $S(R=0.30)$ \\
\hline 130 & 0 & 0 & 0 & 0 & 0 & 0 & 0 & 0 & & \\
180 & 1 & 0 & 0 & 0 & 0 & 0 & 0 & $0.143 \pm 0.132$ & 37,606 & 3,993 & 926 \\
230 & 1 & 1 & 1 & 0 & 1 & 0 & 0 & $0.571 \pm 0.187$ & 4,941 & 550 & 135 \\
280 & 1 & 1 & 1 & 1 & 1 & 1 & 1 & $1 \pm 0.00481$ & 259 & 57 & 21 \\
330 & 1 & 1 & 1 & 1 & 1 & 1 & 1 & $1 \pm 0.00481$ & 259 & 57 & 21 \\
\hline
\end{tabular}

Variation in occupancy rate Occupancy rate increased significantly with elevation (close to $\mathrm{P}=\mathrm{O}$ at $130 \mathrm{~m}$ and $\mathrm{P}=1$ above $280 \mathrm{~m}$; Table 2). The lower altitudinal boundary of the species' presence was $180-230 \mathrm{~m}$ (Table 2). Minimum daily temperatures differed significantly as a function of the elevation (ANOVA, $F=6.9, \mathrm{df}=4, \mathrm{P}<0.01$ ). On average, temperatures were higher at lower elevations, with a difference of c. $0.5^{\circ} \mathrm{C}$ between $130 \mathrm{~m}$ and $330 \mathrm{~m}$.

Abundance of calling males The abundance of calling males varied significantly with elevation (Table 1 ), with a maximum abundance at $230 \mathrm{~m}$ (Fig. 4). No calling males were detected at $130 \mathrm{~m}$, and therefore we did not run simulations for this elevation. For other elevations (180,
230, 280, 330 and $380 \mathrm{~m}$ ) the number of calling males estimated with three, four or five walks was similar to the number estimated with the maximum number (6) of walks $\left(R^{2}=0.97,0.98\right.$ and 0.99, respectively). At $230 \mathrm{~m}$ the mean number of males per site was 12.4. Considering that the area of detection was a circle of radius c. $15 \mathrm{~m}$ around the observer, the estimated density was 1.76 calling males per $100 \mathrm{~m}^{2}$. For both patches the best capture-recapture model was the constant model $(\mathrm{M}(\mathrm{o})$, i.e. with a constant individual probability of capture), which provided an abundance estimate of $10 \pm \mathrm{SD} 0.3$ males for the $600 \mathrm{~m}^{2}$ patch (corresponding to a density of 1.7 calling males per $100 \mathrm{~m}^{2}$ ) and $22 \pm \mathrm{SD} 1.17$ males for the $1,350 \mathrm{~m}^{2}$ patch (corresponding to a density of 1.6 calling males per $100 \mathrm{~m}^{2}$ ). 


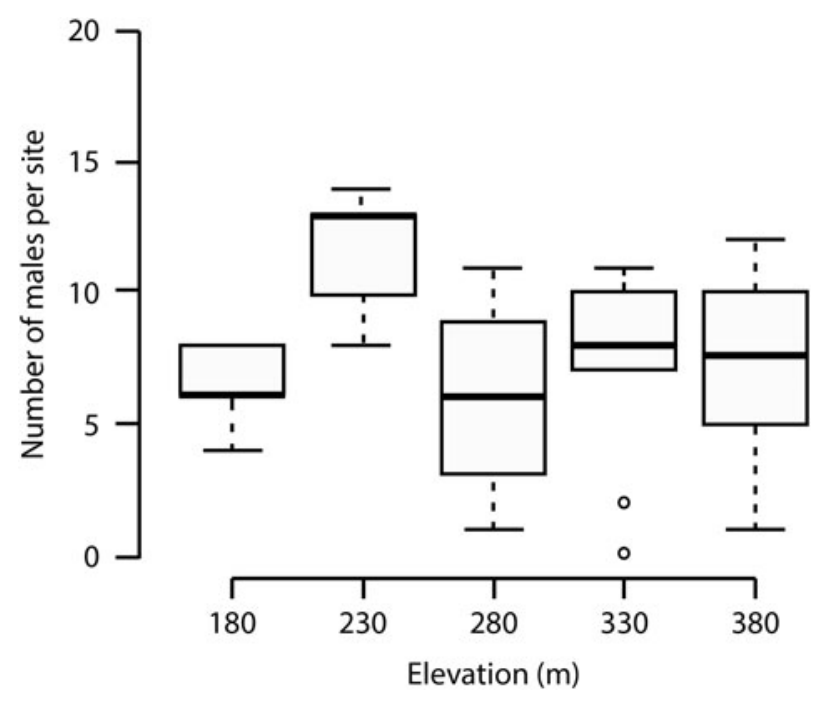

FIG. 4 Number of calling males per site at each elevation surveyed. The bottom and top portions of the boxes represent the first and third quartiles, respectively, and the band between represents the median. The whiskers indicate the limits that include $95 \%$ of the data. Points represent outliers.

Power analysis Given a detection probability of 0.882 at dusk during the rainy season (sessions 1-3), only two detection events $(K=2)$ were required to determine species presence at an occupied site with a probability of 0.95. Even considering $K=2$ for a monitoring protocol, the number of sites necessary to detect a limited decline ( $R=5 \%$ ) would be too high to be implemented ( 259 sites at 280 and $330 \mathrm{~m}, 37,606$ sites at $180 \mathrm{~m}$; Table 2). The number of sites needed to detect population declines of 15 or $30 \%$ was reasonable for elevations $>280 \mathrm{~m}(57$ and 21 sites, respectively; Table 2).

\section{Discussion}

\section{A species threatened by climate change}

Amphibian population declines and species extinctions began to be noted in the early 1980s (Houlahan et al., 2000). Since then, amphibian decline has come to the forefront of the biodiversity crisis. However, reliable estimates of the extent of the decline of amphibians are limited by several factors, particularly in the tropics (Collins \& Halliday, 2005). Firstly, amphibian taxa are still being described at a high rate (Glaw \& Köhler, 1998), which implies that species loss quantified as a percentage of described species is underestimated in many parts of the world (Fouquet et al., 2007). Secondly, many tropical species are rare or elusive, and therefore long-term monitoring programmes must be designed carefully to detect population changes or distributional shifts reliably (Courtois et al., 2013). We intended to design a monitoring programme for $P$. espedeus before any putative decline is detected.
Ecological niche modelling indicates that the distribution of suitable environmental conditions for $P$. espedeus is small. It matches the known distribution of the species well: the highest massifs of the easternmost part of the Guiana Shield. The range of $P$. espedeus is therefore likely to be $<20,000 \mathrm{~km}^{2}$, and fewer than 10 populations are known. Thus, if a decline were observed $P$. espedeus would meet IUCN criteria for categorization as Vulnerable. The environmental suitability projected for 2070 suggests that the extinction of the species is a possibility. The model predictions (an increase in annual temperature and a decrease in rainfall by 2070) suggest that the existing conditions at locations where $P$. espedeus is present will change and there will be no suitable habitat for the species. These predictions need to be considered with caution because they are based on only a few occurrences, assume that the present realized niche of the species matches its fundamental niche (Soberón \& Nakamura, 2009), and do not take into account any possible physiological plasticity of the species. Furthermore, models were based on coarse-resolution bioclimatic layers but microhabitat site selection can buffer climate, limiting the impact of climatic variation. Ecological niche models based on macroclimatic variables could thus lead to overly pessimistic results (Scheffers et al., 2014).

The lower elevation at which $P$. espedeus occurs is $180-280$ $\mathrm{m}$, indicating that its distribution is bound by subtle variations in environmental conditions. Although it is difficult to establish causal linkages between environmental factors and species distribution (Walther et al., 2002), a set of abiotic parameters, including temperature (our results indicate a mean difference of $\mathrm{c} .0 .5^{\circ} \mathrm{C}$ from $130 \mathrm{~m}$ to $330 \mathrm{~m}$ ), are likely to play a fundamental role in bounding the distribution of $P$. espedeus. Niche modelling analysis identifies temperature and precipitation in the driest quarter as the most important factors explaining the distribution of the species. Such a pattern of narrow altitudinal distribution appears to be frequent in the genus Pristimantis, especially in the Andean species (Lehr \& Duellman, 2009), and suggests that this species could be particularly vulnerable to climate change. A slight temperature increase $\left(1^{\circ} \mathrm{C}\right.$ during $\left.1975^{-2000}\right)$ was implicated in the population decline of several Eleutherodactylus spp. in El Yunque, Puerto Rico (Burrowes et al., 2004). Climate change is expected to result not only in a temperature increase but also in decreased precipitation, which would probably also influence any decline of $P$. espedeus, as has been the case for other tropical mountain species (Pounds et al., 1999). Moreover, there is evidence of drought-related decline and increased vulnerability to the pathogenic fungus Batrachochytrium dendrobatidis in Eleutherodactylus species (Longo \& Burrowes, 2010), and the prevalence of the fungus in the $P$. espedeus population should be assessed as soon as possible, as it is known to occur in French Guiana (Courtois et al., 2012). An efficient monitoring protocol is 
therefore needed for early detection of a decline in the distribution or abundance of the species.

\section{Monitoring a climate-driven decline}

Our study provides some insights for implementing a monitoring programme for $P$. espedeus. Using passive acoustic recorders the probability of detection of $P$. espedeus during the rainy season is high $(\mathrm{P}=0.8)$, implying that two recording sessions are sufficient to estimate occupancy rates for the species and, therefore, it would be feasible to monitor a large number of sites each year. Moreover, power analysis indicates that, given the high occupancy rate at altitudes $\geq 280 \mathrm{~m}$, the number of sites necessary to detect a decline in occupancy rate is moderate (e.g. 57 sites to detect a $15 \%$ decline with a power of o.8). Detecting a shift in altitudinal distribution (i.e. detecting a decline in occupancy rate at the lower elevation limit), however, would be more difficult given the lower occupancy rates below $230 \mathrm{~m}$. The calling activity of P. espedeus is limited to dawn and dusk, with peaks of activity during $06.15-06.30$ and $18.30-18.45$ and with more activity at dusk. Such patterns of crepuscular activity are typical in many tropical anurans, particularly Pristimantis (Lehr \& Duellman, 2009). The physiological determinant of this calling phenology is still debated (Lüddecke et al., 2000) but it is an important parameter for a monitoring protocol because it defines a narrow temporal window when detection is likely.

Estimates of population density based on audio transects and repeated count data were similar to those based on the capture-mark-recapture protocol (1.76 and 1.65 calling males per $100 \mathrm{~m}^{2}$, respectively). This suggests that repeated counts can provide reliable estimates for this species, which can be used to track changes in male abundance over time. Three detection events during the breeding season are sufficient to obtain a reliable estimate of the number of calling males. Repeated counts have advantages over capturemark-recapture: they are less invasive, and a wider area can be monitored (Rödel \& Ernst, 2004). They have provided evidence of a decline in Eleutherodactylus gryllus (Puerto Rico), from 3.0 to 0.5 calling males per $100 \mathrm{~m}^{2}$ during 1990-2001 (Burrowes et al., 2004). Density estimation based on number of individuals counted (either through repeated counts or capture-mark-recapture) may be biased because individuals may not reside permanently in the area sampled (Kéry, 2010). The magnitude of the discrepancy depends on the dispersal ability of the species and the time frame of the survey protocol (Kéry, 2010). As the biology of $P$. espedeus suggests low dispersal ability and as our study was conducted over a short time period, such bias was probably limited.

The combination of ecological niche modelling with future climate projections is a useful tool to assess population vulnerability to climate change (Lemes \& Loyola, 2013; Nori et al., 2013). However, testing such predictions is difficult because it requires gathering distribution and abundance data over a long time period. We propose that a standard monitoring protocol for P. espedeus should include (1) 60 recording sites to track changes in occupancy rates at $280 \mathrm{~m}$ (the lowest elevation where occupancy rate is equal to one), with two detection events per year, (2) six audio transects for annual repeated counts, with three walks per transect in good weather conditions, and (3) monitoring of Batrachochytrium dendrobatidis prevalence in the population. We are beginning to implement this monitoring protocol in the northernmost populations (Nouragues, Trinite and Grande Montagne Tortue) of P. espedeus, where reliable climatic data (temperature, hygrometry and precipitation) are available, to identify links between potential population changes and changes in environmental conditions.

\section{Acknowledgements}

This project was conducted at the Nouragues Biological Research Station within the Nouragues Natural Reserve. We thank Valentine Alt, Wemo Betian, Patrick Chatelet and Benoit Villette for help in the field. We thank C.E.T. Paine, Stefan Lötters and one anonymous reviewer for their comments. This work has benefited from two Investissement d'Avenir grants of l'Agence Nationale de la Recherche (AnaEE-S: ANR-11-INBS-0oo1 and CEBA: ANR-10-LABX-25-01) and from technical and human support from USR 3456 through the use of the Nouragues research station.

\section{References}

Aкаiкe, H. (1974) A new look at the statistical model identification. IEEE Transactions on Automatic Control, 19, 716-723.

Blaustein, A.R. \& Wake, D.B. (1990) Declining amphibian populations: a global phenomenon? Trends in Ecology \& Evolution, 5, 203-204.

Bongers, F., Charles-Dominique, P., Forget, P.-M. \& Théry, M. (eds) (2001) Nouragues: Dynamics and Plant-Animal Interactions in a Neotropical Rainforest. Springer Science+Business Media, Dordrecht, Netherlands.

Buckley, L.B., Hurlbert, A.H. \& Jetz, W. (2012) Broad-scale ecological implications of ectothermy and endothermy in changing environments. Global Ecology and Biogeography, 21, 873-885.

Burrowes, P.A., Joglar, R.L. \& Green, D.E. (2004) Potential causes for amphibian declines in Puerto Rico. Herpetologica, 6o, 141-154.

Cahill, A.E., Aiello-Lammens, M.E., Fisher-Reid, M.C., Hua, X., KarANEWSKY, C.J., RYu, H.Y. et al. (2013) How does climate change cause extinction? Proceedings of the Royal Society B, 280, 20121890.

Charif, R., Ponirakis, D. \& Krein, T. (2006) Raven Lite 1.o User's Guide. Cornell Laboratory of Ornithology, Ithaca, USA.

Collins, J.P. \& Halliday, T. (2005) Forecasting changes in amphibian biodiversity: aiming at a moving target. Philosophical Transactions of the Royal Society B, 360, 309-314.

Courtois, E.A., Devillechabrolle, J., Dewynter, M., Pineau, K., Gaucher, P. \& Chave, J. (2013) Monitoring strategy for eight 
amphibian species in French Guiana, South America. PLoS ONE, 8 (6), e67486.

Courtois, E.A., Pineau, K., Villette, B., Schmeller, D.S. \& Gaucher, P. (2012) Population estimates of Dendrobates tinctorius (Anura: Dendrobatidae) at three sites in French Guiana and first record of chytrid infection. Phyllomedusa, 11, 63-70.

Dawson, T.P., Jackson, S.T., House, J.I., Prentice, I.C. \& Mace, G. M. (2011) Beyond predictions: biodiversity conservation in a changing climate. Science, 332, 53-58.

Duarte, H., Tejedo, M., Katzenberger, M., Marangoni, F., Baldo, D., Beltrán, J.F. et al. (2012) Can amphibians take the heat? Vulnerability to climate warming in subtropical and temperate larval amphibian communities. Global Change Biology, 18, 412-421.

Elith, J., Phillips, S.J., Hastie, T., Dudík, M., Chee, Y.E. \& Yates, C.J. (2011) A statistical explanation of MaxEnt for ecologists. Diversity and Distributions, 17, 43-57.

Fiske, I.J. \& ChANDler, R.B. (2011) unmarked: An R package for fitting hierarchical models of wildlife occurrence and abundance. Journal of Statistical Software, 43, 1-23.

Foden, W.B., Butchart, S.H.M., Stuart, S.N., Vié, J.-C., AкÇAkaya, H.R., Angulo, A. et al. (2013) Identifying the world's most climate change vulnerable species: a systematic trait-based assessment of all birds, amphibians and corals. PLoS ONE, 8(6), e65427.

Fouquet, A., Gilles, A., Vences, M., Marty, C., Blanc, M. \& Gemmell, N.J. (2007) Underestimation of species richness in neotropical frogs revealed by $\mathrm{mtDNA}$ analyses. PLoS ONE, 2(10), e1109.

Fouquet, A., Martinez, Q., Courtois, E.A., Dewynter, M., Pineau, K., Gaucher, P. et al. (2013) A new species of the genus Pristimantis (Amphibia, Craugastoridae) associated with the moderately elevated massifs of French Guiana. Zootaxa, 3750, 569-586.

Franklin, J. (2009) Mapping Species Distributions: Spatial Inference and Prediction. Cambridge University Press, Cambridge, UK.

Glaw, F. \& Köhler, J. (1998) Amphibian species diversity exceeds that of mammals. Herpetological Review, 29, 11-12.

Guillera-Arroita, G. \& Lahoz-Monfort, J.J. (2012) Designing studies to detect differences in species occupancy: power analysis under imperfect detection. Methods in Ecology and Evolution, 3, 860-869.

Guillera-Arroita, G., Ridout, M.S. \& Morgan, B.J. (2010) Design of occupancy studies with imperfect detection. Methods in Ecology and Evolution, 1, 131-139.

Hedges, S.B., Duellman, W.E. \& Heinicke, M.P. (2008) New World direct-developing frogs (Anura: Terrarana): molecular phylogeny, classification, biogeography, and conservation. Zootaxa, 1737, 1-182.

Houlahan, J.E., Findlay, C.S., Schmidt, B.R., Meyer, A.H. \& Kuzmin, S.L. (2000) Quantitative evidence for global amphibian population declines. Nature, 404, 752-755.

Joseph, L.N., Field, S.A., Wilcox, C. \& Possingham, H.P. (2006) Presence-absence versus abundance data for monitoring threatened species. Conservation Biology, 20, 1679-1687.

KérY, M. (2010) Introduction to WinBUGS for Ecologists. Academic Press, Burlington, USA.

Lehr, E. \& Duellman, W.E. (2009) Terrestrial-Breeding Frogs (Strabomantidae) in Peru. Natur und Tier Verlag, Münster, Germany.

Lemes, P. \& Loyola, R.D. (2013) Accommodating species climate-forced dispersal and uncertainties in spatial conservation planning. PLoS ONE, 8(1), e54323.

Longo, A.V. \& Burrowes, P.A. (2010) Persistence with chytridiomycosis does not assure survival of direct-developing frogs. EcoHealth, 7, 185-195.

Lüddecke, H., Amézquita, A., Bernal, X. \& Guzmán, F. (2000) Partitioning of vocal activity in a Neotropical highland-frog community. Studies on Neotropical Fauna and Environment, 35, 185-194.
MacKenzie, D.I., Nichols, J.D., Lachman, G.B., Droege, S., Royle, J.A. \& LANGTimm, C.A. (2002) Estimating site occupancy rates when detection probabilities are less than one. Ecology, 83, 2248-2255.

Moss, R.H., Edmonds, J.A., Hibbard, K.A., Manning, M.R., Rose, S.K., VAN VUUREN, D.P. et al. (2010) The next generation of scenarios for climate change research and assessment. Nature, 463 , 747-756.

Nori, J., Lescano, J.N., Illoldi-Rangel, P., Frutos, N., Cabrera, M.R. \& Leynaud, G.C. (2013) The conflict between agricultural expansion and priority conservation areas: making the right decisions before it is too late. Biological Conservation, 159, 507-513.

Parmesan, C. \& Yohe, G. (2003) A globally coherent fingerprint of climate change impacts across natural systems. Nature, 421, 37-42.

Pearson, R.G., Raxworthy, C.J., Nakamura, M. \& Peterson, A.T. (2007) Predicting species distributions from small numbers of occurrence records: a test case using cryptic geckos in Madagascar. Journal of Biogeography, 34, 102-117.

Phillips, S.J., Anderson, R.P. \& Schapire, R.E. (2006) Maximum entropy modeling of species geographic distributions. Ecological Modelling, 190, 231-259.

Pounds, J.A., Bustamante, M.R., Coloma, L.A., Consuegra, J.A., Fogden, M.P.L., Foster, P.N. et al. (2006) Widespread amphibian extinctions from epidemic disease driven by global warming. Nature, 439, 161-167.

Pounds, J.A., Fogden, M.P.L. \& Campbell, J.H. (1999) Biological response to climate change on a tropical mountain. Nature, 398 , 611-615.

R Development Core Team (2014) R: A Language and Environment for Statistical Computing. R Foundation for Statistical Computing, Vienna, Austria.

RÖDEL, M.-O. \& ERnst, R. (2004) Measuring and monitoring amphibian diversity in tropical forests. I. An evaluation of methods with recommendations for standardization. Ecotropica, 10, 1-14.

Royle, J.A. (2004) N-mixture models for estimating population size from spatially replicated counts. Biometrics, 6o, 108-115.

Scheffers, B.R., Edwards, D.P., Diesmos, A., Williams, S.E. \& Evans, T.A. (2014) Microhabitats reduce animal's exposure to climate extremes. Global Change Biology, 20, 495-503.

Sheldon, K.S., YAnG, S. \& Tewksbury, J.J. (2011) Climate change and community disassembly: impacts of warming on tropical and temperate montane community structure. Ecology Letters, 14, 1191-1200.

SNYDER, G.K. \& WeAthers, W.W. (1975) Temperature adaptations in amphibians. The American Naturalist, 109, 93-101.

Soberón, J. \& NAKAmura, M. (2009) Niches and distributional areas: concepts, methods, and assumptions. Proceedings of the National Academy of Sciences of the United States of America, 106, 1964419650.

Stanley, T.R. \& Burnham, K.P. (1999) A closure test for time-specific capture-recapture data. Environmental and Ecological Statistics, 6, 197-209.

Stuart, S.N., Chanson, J.S., Cox, N.A., Young, B.E., Rodrigues, A.S.L., Fischman, D.L. \& Waller, R.W. (2004) Status and trends of amphibian declines and extinctions worldwide. Science, 306, $1783-1786$.

Sunday, J.M., Bates, A.E. \& Dulvy, N.K. (2011) Global analysis of thermal tolerance and latitude in ectotherms. Proceedings of the Royal Society B, 278, 1823-1830.

Thomas, C.D., Franco, A.M.A. \& Hill, J.K. (2006) Range retractions and extinction in the face of climate warming. Trends in Ecology \& Evolution, 21, 415-416. 
Travis, J.M.J. (2003) Climate change and habitat destruction: a deadly anthropogenic cocktail. Proceedings of the Royal Society B, 270 , 467-473.

Walther, G.-R., Post, E., Convey, P., Menzel, A., Parmesan, C., B EевеE, T.J.C. et al. (2002) Ecological responses to recent climate change. Nature, 416, 389-395.

WARREN, D.L. (2012) In defense of 'niche modeling'. Trends in Ecology \& Evolution, 27, 497-500.

White, G.C. \& Burnham, K.P. (1999) Program MARK: survival estimation from populations of marked animals. Bird Study, 46, S120-S139.

\section{Biographical sketches}

Elodie Courtois works on amphibian monitoring and conservation and was responsible for setting up the meteorological station and environmental sensors at the Nouragues research station. Elodie Michel and Quentin Martinez are interested in tropical amphibians. Kevin PineAu and MAëL DeWYNTER are interested in animal conservation and the management of protected areas in French Guiana. Gentile Ficetola is interested in the application of landscape ecology, evolutionary ecology and landscape genetics to conservation action. Antoine Fouquet is interested in the phylogeography, biogeography and evolution of tropical frogs. 